


\section{La Universidad Santo Tomás: un modelo de universidad de Estudio General}

Miguel Moreno Lugo*

\section{Introducción}

En este capítulo se quiere responder, en pocas páginas, a cuál es el modelo de Universidad que después de 436 años sigue siendo fiel al carisma que la originó.

Las constituciones de los estudios dominicos que tenemos a nuestro alcance se refieren exclusivamente a la formación de los frailes; esa condición impone un desafío adicional a este trabajo en la medida en que nuestra tarea consiste en hablar específicamente de la Universidad como Institución. Por lo tanto, no se referirá este capítulo a la formación de frailes, sino a la Universidad que ellos crearon como Universidad dominicana, de Estudio General, católica y tomista.

* Licenciado en Filosofía y Letras, Universidad Santo Tomás, Bogotá; Maestría en Filosofía Latinoamericana, Universidad Santo Tomás, Bogotá. Docente del Departamento de Humanidades y Formación Integral, Universidad Santo Tomás, Bogotá. 
De esta manera, y en razón a las limitaciones que el libro exige para este capítulo, en primer lugar, se hará una muy sucinta revisión de los modelos de universidad española que impactaron notablemente al nuevo mundo; en segundo lugar, hablaremos del modelo de colegio-universidad que se dio en la colonia; finalmente, mencionaremos algunos apuntes que permiten comprender cómo se desarrolló el modelo de Estudio General en la tomística hasta su restauración en 1965.

\section{Modelos universitarios en España: salamantino y alcaíno}

Si hay dos coordenadas que deben considerarse para pensar a Occidente son, de una parte, la cristiandad y, de otra, el fenómeno universitario. Esto en razón a que la primera le dio una idea de orden social y la segunda se refiere a cómo la institución universitaria permitió desarrollar muchas implicaciones de la ontología que el cristianismo exigía para ser posible su proyecto de orbe cristiano. Es así como los estudios de gramática, teología, derecho, medicina y artes moldearon a Occidente de acuerdo al deber ser que la religión católica y la monarquía cristiana imponían.

La institución de la enseñanza era una práctica más o menos común, poco organizada, pero que progresivamente se iba expandiendo desde Europa. Previamente, el continente había conocido la experiencia de las escuelas y, en especial, las de traductores de los saberes árabes. La práctica era reconocida por diferentes nombres, según la época y el lugar, pero en general los nombres más recurrentes eran los de scholas, escuelas, academia, estudio, studium, studium solemne, studium generale y universitas. En España, el nombre de Estudio General fue el más recurrente, y ya en 1225 se le adhirió a Salamanca. Estas instituciones tenían privilegios reales, del papado o ambos; su principal instrumento de poder era el monopolio para 
otorgar grados que acreditaban a sus egresados para ejercer cargos públicos y reconocimiento social a través de la licentia docendi $i^{1}$. Sin embargo, era el papa o el emperador quienes podían otorgar a quien se graduaba el ius ubique docendi ${ }^{2}$. Este título determinaba hasta qué punto un studium tenía la categoría de Escuela Universitaria (Santiago Otero, 1996), ya que permitía enseñar en cualquier lugar de la cristiandad. Hay que tener en cuenta que Bolonia, en la Facultad de Derecho, y París, en Teología, tenían el atributo de que sus licentia docendi facultaban a sus egresados para enseñar en cualquier Estudio General.

Sea como fuere, desde su concepción hasta hoy, es en las partidas de Alfonso X el Sabio donde se encuentra la mejor definición de Estudio o Universidad que pueda encontrarse para referirse a la institución desde esa época hasta casi el siglo XIX, debido a que con los modelos napoleónicos, humboldtianos, de politécnicos y de universidades empresariales esta naturaleza original cambió:

Estudio es ayuntamiento de maestros et de escolares que es fecho en algunt logar con voluntad et con entendimiento de aprender los saheres: et son dos maneras dél; la una es á que dicen estudio general en que ha maestros de las artes, así como de gramática, et de lógica, et de retórica, et de arismética, et de geometria, et de musica et de astronomia, et otmsi en que ha maestros de decretos et señores de leyes: et este estudio debe seer establescido por mandato de papa, ó deemperador ó de rey. La segunda manera es á que dicen estudio parartida que quier tanto decir como cuando algunt maestro amuestra en alguna villa apartadamente

1 Según el canon 18 del tercer concilio de Letrán.

2 La expresión significa derecho a enseñar en cualquier parte y se usó por primera vez por el papa Gregorio IX en la Universidad de Toulouse en 1233. 
á pocos escolares; et tal como este puede mandar facer perlado ó concejo de algunt logat. (Barcala Muñoz, 1985)

En esa medida, podemos comprender que después del renacimiento carolingio surgieron dos modelos de casas de estudio:

La forma de Universitas Scholarium (comunidad o gremio de estudiantes) y posteriormente Universitas Magistroum (comunidad o gremio de maestros). Este señalamiento nos parece importante, ya que, en el primer caso, los grupos organizados de estudiantes contrataban maestros para que les impartieran formación básica y formación profesional en medicina, derecho o teología. Los estudiantes regían la Universitas y el rector era un estudiante; el prototipo fue la Universidad de Bolonia. En el segundo caso, los maestros ofrecían los mismos servicios a los jóvenes, pero eran los docentes quienes regían y el rector era un maestro; en este caso, el prototipo fue la Universidad de París. (Arredondo Vega, 2011, p. xx)

Así pues, siguiendo a Arredondo, vemos que el modelo universitario buscaba cumplir con tres características fundamentales: primero, que estuviera abierto a estudiantes de cualquier parte; segundo, que se impartiera enseñanza superior en facultades y, finalmente, que existieran muchos maestros para enseñar las diferentes cátedras (Santiago-Otero, 1996). Sin embargo, estos rasgos comunes se derivan de los modelos universitarios que había en la Edad Media.

En efecto, para Peset y García (Peset \& García Trobat, 2013), por su posición geográfica, el modelo boloñés estaba a medio camino entre el papado y el imperio. Muchos de sus miembros eran güelfos que formaban sociedades con algún maestro que les enseñara y le pagaban por medio de una colecta para adquirir algún grado o título, pero el ayuntamiento empezó a ofrecer los cursos 
y a atacar a los güelfos que optaban por esta opción de estudios. Federico I, Barbarroja, los defiende, pero los deja bajo jurisdicción de un obispo - tal como lo había hecho Justiniano en la escuela de derecho de Beirut. Los estudiantes de cada nación nombraban sus procuradores y consiliarios. Los profesores eran designados por los escolares y los rectores; estos últimos señalaban las materias y puntos que se estaría bien explicar. Los doctores que fungían como examinadores para los graduados no hacían parte de la universidad. Por su postura güelfa, Honorio III nombró al archidiácono de la catedral de Bolonia para que otorgara solemnemente los grados.

El segundo modelo que nos refieren los autores es el modelo parisino, el cual surge en torno a Nôtre Dame, principalmente formada por maestros de artes de diferentes regiones; un delegado de cada nación: la isla de Francia, la normanda, la picada y la inglesa elegían al rector. Surgió como una corporación universitaria del enfrentamiento entre maestros y estudiantes con el maestrescuela de la catedral, que, por sus exigencias en la concesión de grados, pretendía también que le juraran obediencia con la pérdida de autonomía y privilegios. Inocencio III, al optar por la corporación de maestros, le dio la fisonomía que la caracterizaría.

Finalmente, el tercer modelo provino de Oxford. En este modelo no existía la figura de rector, sino que había un canciller designado por el obispo, que fue perpetuo después del siglo XV. En las universidades inglesas, las residencias y los colegios tuvieron mucha importancia; por lo general fueron fundados y dotados por prelados o nobles, de manera que eran más autónomos; el rector del colegio era nombrado por los profesores o por el rey directamente. También existía la figura de un tutor, nombrado por el rector, que ayudaba y dirigía el estudio de los alumnos. La enseñanza se concentra en los colegios, mientras que la universidad se interesaba más en los grados.

En síntesis, podemos decir que de una parte estaba el modelo de Bolonia, que se caracterizaba porque el rector era elegido 
entre los colegas y, por otro, el modelo de París, en el que el rector era impuesto por una autoridad (el rey o un obispo). Hernández y Casado nos refieren que la máxima evolución de esos modelos fue la fórmula colegio-universidad, Convento-Universidad o Seminario-Universidad; estos se destacan por el carácter colegiado que imprimieron a su vida académica y se constituyeron en el modelo que más se implantó en América. Los colegios-universidad (menor) de San Antonio de Portaceli de Sigüenza (1489), el Mayor de San Ildefonso (1543) y la Universidad de Alcalá de Henares (1499) fueron las instituciones más destacadas que acogieron este modelo. Por lo general, la universidad se alojaba en un colegio o un convento previamente fundado; en razón de ello, los cargos como los de procuradores y consiliarios eran designados por los colegiales a través del voto (Hernández González \& Casado Arboniés, 2004).

En un primer momento, estas fundaciones fueron patrocinadas por personas que hicieron las veces de sus protectores y patrocinadores; el fundador, por lo general un mecenas, un religioso o un ayuntamiento tenía que alojar un cierto número de estudiantes becados, debía adoptar algunas cátedras, pedir las licencias de autoridad secular y eclesiástica, pedir autorización para otorgar grados, conseguir los edificios y procurar cómo sostener a través de las rentas toda la organización. Sin embargo, para mediados del siglo Xv, la distinción entre universitas y studium generale se perdió. Prácticamente eran sinónimos. A veces la diferencia radicaba más en el modelo de universidad que se ostentaba, ya que esa condición podría indicar la orientación del instituto: París en teología, Bolonia en leyes y Salerno en medicina. Bolonia proporcionaba el modelo de universidad de estudiantes, París el modelo de universidad de maestros (Magallón Ibarra, 2002). Cada nueva universidad debía inscribirse en un modelo de universidad, como fue el caso de la Tomística, que al ser fundada como Convento-Universidad fue alcaína, pero después fue reconocida como hija de Salamanca y "la corona dispuso que en cuanto a colación de grados 
se atuviera a las prácticas de Lima, hija legítima de Salamanca, cuya imagen reprodujo en sus leyes y costumbre" (Rodríguez Cruz, 2008, p. 83). Ahora bien, para señalar cómo los grandes modelos universitarios heredados por España se implantaron en América debemos tener en cuenta, por lo menos, los aspectos que se mencionan a continuación.

Primero, España, desde siempre, ha sido un caso especial en la historia de Europa. En algunos momentos, su distancia con grandes centros de desarrollo como Roma, Alejandría o Estambul hicieron de ella una región rezagada en múltiples aspectos científicos y culturales. De igual modo, su cercanía con África la permeó de muchas costumbres y usos mediterráneos que afectaron su semblante. Finalmente, esa posición geográfica fue la que le dio la oportunidad de encontrar un Nuevo Mundo, siendo ella la principal transmisora del orden europeo (cultura, religión, lengua, política, etc.); es decir, América conoció a Europa a través de los ojos del español. Por último, su tibetanismo contrarreformista (Marquínez Argote, 2001) la aisló de muchos desarrollos del mundo moderno, de aquí que en la Nueva Granada se haya desarrollado la segunda escolástica.

Segundo, en algún momento de la historia no fue tan clara la línea divisoria de un modelo a otro. Para algunos, Estudio General o Universidad eran sinónimos; para otros, el primero fue el paso previo para ser universidad. En tercer lugar, se refiere a que en muchos casos hubo Estudios Generales o corporaciones de maestros y estudiantes con la voluntad de enseñar y aprender que "no tenían su documentación al día" (Santiago-Otero, p. 49); es decir, se elevaba solicitud a la Corona o al Papado y la respuesta tardaba años. En otras ocasiones, llegaba una de las dos licencias, pero no había ninguna institución funcionando y, en otros casos, con ser declarada pontificia era suficiente. Igualmente, en ocasiones la suficiencia se obtenía con el Pase Regio; ejemplo de estas situaciones fue la larga disputa entre jesuitas y dominicos en la Nueva 
Granada o en Santo Domingo entre las universidades de Santiago de la Paz y la Santo Tomás. En algunos momentos, si no se tenían los dos documentos para afirmar que era una institución regia y pontificia, no se reconocía su legitimidad (Woods, 2005)². El cuarto aspecto se refiere a que, debido a la manera particular en que se dieron los hechos en América en los ámbitos políticos, económicos y sociales, la creación, funcionamiento, cierres y restauraciones de universidades fue más allá de un aspecto puramente académico, un juego de intrigas e intereses particulares donde se defendían dispositivos de poder que modificaron la sociedad y las estructuras coloniales en muchos aspectos.

Teniendo esas consideraciones, podemos afirmar que la universidad que llega a nosotros es heredera del modelo universitario de Bolonia, el cual es incorporado en España por el Estudio General de Palencia, pero que una vez extinto, Coímbra, Salamanca y Alcalá fueron los que rigieron los destinos académicos de la península, de los cuales, en este texto, solo se hará referencia a Salamanca y a Alcalá por su influencia en América.

Sobre el modelo de Alcalá

Alcalá debe su fundación al rey Sancho IV, en 1293, como Estudio General, pero solo hasta el año de 1499 el cardenal Cisneros tomó como modelo el Colegio-Universidad de San Antonio de Portaceli, en Sigüenza para diseñar Alcalá; de esta manera, ordenó en sus estatutos un esquema completamente diferente al de Salamanca.

De acuerdo con Manuel Hernández (Hernández González \& Casado Arboniés, 2004), podemos decir que Alcalá se fundó

3 Ochentaiuna universidades se establecieron por el tiempo de la Reforma. De estas, 33 tenían un documento papal, 15 un decreto real o imperial, 20 tenían ambos y 13 no tenían ninguno. 
siguiendo el modelo parisino en cuanto a la formación teológica. La Universidad estaba rodeada de colegios. El rector se encargaba de los miembros del colegio; él y sus consiliarios aceptaban a los estudiantes que pagaban su estancia, nombraban el notario y el receptor, y un colegial era encargado del dinero y de las cuentas. Las grandes decisiones eran sometidas al rector y aprobadas por los colegiales. Los colegiales decidían quién entraba al colegio. El aspirante debía tener mínimo veinte años, preferiblemente se admitían personas pobres, solteras, que no pertenecieran al clero regular, que no fueran de la región de Alcalá, que fuera limpio de sangre, que en las informaciones se pudiera comprobar que en la genealogía familiar no hubo moros, ni judíos, ni juzgados por la inquisición y que los padres tuvieran un buen nombre y un trabajo destacado. Por su parte, los estudiantes escogían a los capellanes.

Carlos Tünnermann brinda otros detalles de cómo funcionaba una universidad que seguía el modelo de Alcalá: el modelo alcaíno fue el preferido por las órdenes religiosas porque el modelo de convento-universidad favoreció el concepto de formación y lo vinculó a una práctica educativa destinada para las élites. Esto, en razón a que vinculaba la idea de formación dentro de un carisma para la vida profesional de los dirigentes, es decir, su actividad profesional estaba fuertemente influenciada por la visión de mundo que el carisma ostentaba; por esta connotación se dice que de Alcalá devienen las universidades privadas. Además, por ser un modelo de convento-universidad, favoreció la formación en teología como télos de las demás ciencias, como se preconizaba desde la Edad Media, y en la que concebía que el resto de las ciencias estaban supeditadas a la Teología.

En muchas universidades que adoptaron este modelo, la colegiatura se escogía entre los frailes dirigentes de la institución, no entre el conjunto de los estudiantes; así que podría hablarse de una formación conventual en donde el rector era a veces el mismo provincial. En este modelo, los colegios tuvieron más importancia que 
en otras instituciones (Tünnermann Bernheim, 1996). Alcalá reformó las administraciones, el gobierno y el modelo de enseñanza. Podemos conocer rasgos de la vida universitaria en el Guzmán de Alfarache y en el episodio de La tía fingida de Cervantes (Jiménez Moreno, 1996). Este modelo fue asumido por la Tomística y la academia Javeriana, aunque en sus constituciones obtienen los privilegios de Salamanca; otras universidades con este modelo fueron la Universidad de Santiago de la Paz, en Santo Domingo, fundada en 1558 y extinguida en 1767; la de San Fulgencio de Quito, fundada en 1586 y extinguida en 1786; la de Nuestra Señora del Rosario, creada en 1619, funcionó hasta 1738; la de San Miguel, al igual que la anterior, en Santiago de Chile, fundada en 1621 y extinguida en 1769; la de San Ignacio de Loyola en Cuzco, Perú, que existió entre 1621 y 1767; la de San Nicolás de Santafé, Nuevo Reino de Granada, establecida en 1694 y clausurada en 1775; la de San Francisco Xavier de Panamá, que funcionó entre 1749 y 1767 (Tünnermann, 1996).

\section{Sobre el modelo Salamanca}

Este modelo de universidad se inspiró en Bolonia y por ello favoreció los estudios jurídicos; desde temprano, empezó a rivalizar con los grandes centros europeos. Por las circunstancias políticas y sociales de la península, Salamanca se convirtió en referente de catolicidad y fue, si se permite afirmarlo, la universidad al servicio de las instituciones del Estado. Esta fue favorecida por los gibelinos.

Fundada en 1218, estuvo desde muy temprano acompañada por la orden dominicana. Los primeros frailes se habían alojado en el convento de la iglesia de San Juan el Blanco. Al desaparecer este recinto, fueron trasladados a la iglesia de San Esteban en 1256, allí fijaron su centro de estudios casi desde sus comienzos y fundaron en 1299 el Estudio General, el cual albergó la escuela 
teológico-jurídica de los siglos XVI y XVII. El Estudio se incorporó a la universidad de Salamanca, regentando la Facultad de Teología en el siglo xx (Historia de la Facultad de Teología, 2016). Es decir, Salamanca surgió del Estudio en derredor de la escuela catedralicia, tal como ocurrió en París. (Peset Reig, 2015). Sus constituciones fueron ratificadas por Martín V en 1422 (Rodríguez Cruz, 1998).

Esta universidad ha conservado su antigua tradición en sus regímenes ceremoniosos, los ritos de graduación y el modelo de maestro (Migueláñez, 2011). El claustro de profesores era la máxima autoridad académica y podía reformar los estatutos. Al maestrescuela o canciller, por lo general un alto prelado, le correspondían las importantes funciones de juez de los estudios y el otorgamiento de grados; el de rector se preocupaba por la representación universitaria y la supervisión de la docencia - este era un cargo menor al de canciller. El rector era asesorado por el consejo consiliario y por el consejo de diputados que se encargaban de las decisiones y del manejo de recursos económicos. Matricularse en la universidad implicaba el juramento de obediencia licitus et honestis. El papel de la cátedra era fundamental, como era el caso de la Cátedra Prima que ostentó hasta su muerte Francisco de Vitoria. La cátedra se otorgaba mediante oposición, así:

Los estudiantes participaban activamente en los concursos formando bandos en pro y en contra de los candidatos. Exaltados los ánimos menudearon los desórdenes a propósito de estos ejercicios, lo que movió a la Corona a retirar a los estudiantes el derecho de voto. Las oposiciones más reñidas, y que suscitaban mayor expectación, eran las de la Facultad de Teología por las rivalidades entre las órdenes religiosas, interesadas en controlar determinadas cátedras para imponer sus criterios en relación a ciertos puntos de doctrina. Por tratarse de un asunto capaz de producir enconadas controversias, los estatutos reglamentaban 
con prolijidad todo lo referente a estos concursos, a fin de precaver fraudes y sobornos, lo que no siempre lograron. (Tünnermann, 1996, p. 33)

Las facultades de Artes y Teología otorgaban los grados de bachiller, licenciado y maestro; Derecho y Medicina llegaban hasta el grado de doctor. El método de enseñanza se basaba en la lectio y la disputatio. Salamanca dio origen al modelo de universidad pública. Lima y México fueron universidades creadas por iniciativa real y fueron diseñadas en el modelo salamantino hasta en sus más pequeños detalles (Tünnermann, 1996); otras universidades que siguieron este mismo modelo fueron las de Guatemala, Guadalajara, León de Nicaragua, Charcas, Huamanga y Caracas, entre otras (Rodríguez Cruz, 1998).

\section{Sobre el Colegio y la Universidad}

Evidentemente los religiosos aprovecharon el modelo alcaíno y la herencia salamantina para diseñar, a bajo costo, un modelo de centro de estudios que resultara eficiente para sus propósitos: a) la evangelización del Nuevo Mundo, b) el apoyo a ciertas posturas en términos doctrinales y c) mantener el monopolio de la educación. Es de tener en cuenta que:

El clero regular monjes y frailes cumplía un papel notable en los estudios y universidades. Desde antiguo desempeñaban cátedras en muchas de ellas, en especial en las facultades de Teología y en Cánones, en Artes. Pero además erigieron centros propios. Crearon colegios o conventos cerca de las universidades para que sus miembros pudieran acudir a las aulas y graduarse: la orden dominica, San Esteban en Salamanca o San Gregorio en Valladolid. Abrieron otros centros docentes 
para la formación de sus novicios, dando títulos particulares de presentado y maestro, sin validez universitaria. Ahora ven un resquicio para poder tener universidades propias. Usan una fórmula, sencilla, menos costosa: un convento o un colegio o casa de la orden, en donde estudiaban los frailes, se abría a externos y, obtenida la bula de la santa sede, confería grados. Con un coste mínimo quedaban convertidos en universidad o Estudio General. (Peset \& García Trobat, 2013, p. 115)

Con lo anterior, la institución mixta que surgía a manos de los religiosos - particularmente en América- permitía conjugar todos los aspectos anteriormente señalados como una unidad orgánica que se prestaba para ser universidad, para formar en un carisma, para ser casa de estudios de las órdenes, para desplegar un modo de vida muy similar a la vida comunitaria y para desarrollar un currículo especialmente diseñado para las necesidades que la institución asumía como propias y se proponía responder desde su identidad.

De esta manera, franciscanos, jesuitas y dominicos, sobre la base de una institución ya creada, colegio o convento, fundan una universidad. Este ejemplo lo toman de Alcalá, que partió sobre la base de San Antonio de Portaceli de Sigüenza; algunas universidades que siguieron el esquema de Colegio-Universidad, Colegio-Seminario, Convento-Universidad, Estudio-Universidad fueron: Santiago de la Paz, en Santo Domingo (1558), Universidad Tomista, en Santafé (1580), San Fulgencio (1586) en Quito, Nuestra Señora del Rosario (1619) en Santiago, Academia Javeriana en Santafé (1621), Córdoba en Argentina (1621), San Gregorio Magno (1621) en Quito, San Ignacio de Loyola (1621) en Cuzco, San Nicolás (1694) en Santafé y, la jesuita en el Colegio-Seminario de san Francisco (1744) en Popayán (Jiménez Moreno, 1996).

El término "colegio", derivado del latín collegium significa asociación, comunidad, unión, "el hecho de ser colegas". En este 
sentido, se le da al término la significación e interpretación de corporación, sociedad, donde se reúnen gentes que comparten las mismas inquietudes. Así, actualmente, el término colegio se usa para establecer asociaciones profesionales, centros de enseñanza, o residencias de estudiantes. Pero lo cierto es, que en todas y cada una de las aceptaciones que podamos escoger, está la cuestión de comunidad. (Martín Sánchez, 2009, p. 368)

Si bien es cierto que los colegios fueron pensados como residencia de naciones, en donde los estudiantes pudieran ser acogidos y gozaran de ciertos privilegios que una persona corriente no podía tener, también lo es que ser admitido en un colegio legitimaba la blancura para las familias de clase dominante, era el reconocimiento público de su condición de blancos y limpieza de sangre. Esto permitía asegurar controladamente el acceso a cargos públicos "porque vestir una beca en uno de los colegios mayores significaba entrar a formar parte del plan más selecto de la sociedad" (Castro-Gómez, 2005, p. 121). Continuando con Castro-Gómez, podemos referir que para ingresar a la universidad se debían contestar unos cuestionarios conocidos como las informaciones. Después de los Borbones, el interrogatorio y la revisión del árbol genealógico del candidato fue más exigente. El que aspira a ser admitido se asume como perteneciente a una logia, debía prestarse a su consagración y al juramento, un rito con la carga simbólica semejante a la de caballero del rey. Los colegio-universidad fueron creados para formar a las élites, a las clases dirigentes; tal es el caso de la Tomística, cuya primitiva Misión podría plasmarse como sigue:

Universidad "de estudio general" para cultivar y difundir la comprensión tomista de la realidad - forma oficial de comprensión católica en tiempos de "contrarreforma"-, donde pudiesen aprender "letras y virtud" y optar todos los grados académicos, en las facultades autorizadas, "muchas personas de esa tierra", 
particularmente hijos de caciques y descendientes de colonizadores que no podían viajar ni a Méjico ni a Lima, haciéndose competentes para enseñar, ejercer profesiones liberales o acceder a cargos públicos. (USTA, 2004, p. 44)

Como podemos apreciar, los estudiantes de la tomística en Santafé se adecuaban a los perfiles que en la época se exigía, por lo tanto, entre los colegiales surgían afinidades y sentido de identidad y de rivalidad con otros colegiales. En los colegios se estudiaba prácticamente las mismas materias que en la universidad, solo que la universidad atendía más a la enseñanza, mientras que en los colegios se enfatizaba más en la formación de una clase bien disciplinada capaz de regir los destinos de otros. La formación era de carácter confesional, se procuraba formar en los valores cristianos y en las virtudes como camino para reconocer la vocación del hombre a su creador. Así pues, los Colegios se entendían como

Instituciones históricas que, al lado de nuestras gloriosas Universidades [...] tenían como finalidad dar residencia, estudios, alimentos, libros, vestido, tutela y protección a los estudiantes universitarios; y coincidentes con el florecimiento de las Universidades, caminaron a su vera tanto en lo científico como en el sistema disciplinar y pedagógico. Y su último objetivo, en sustancia, era formar una clase directora. (Martín Hernández, 1992, p. 11)

Por clase directora no solo se referían a los españoles o a los españoles nacidos en el Nuevo Mundo, sino también a los manteos, quienes eran jóvenes, hijos de mestizos enriquecidos y que eran reclutados con el fin de cubrir las necesidades de los religiosos. Por lo general, eran admitidos como aspirantes al sacerdocio, eran un grupo étnico aparte dentro de la universidad, no tenían contacto con los estudiantes limpios de sangre y sin mancha de la tierra, no 
podían aspirar a altos cargos directivos, sus ocupaciones eran más bien rezagadas a los cargos medios (Castro-Gómez, 2005, p. 121).

También es cierto que en los colegios (Convento-Estudio) se vivía con el rigor de la formación clerical que la época exigía:

Se controlaba la asistencia a clase (...) La pedagogía, la enseñanza, la educación era el objetivo y la idea central, y en ocasiones la obsesión, en torno a la cual giraba la vida del colegio. Los ejercicios intensos y la religiosidad caracterizaban la vida diaria de un colegio universitario. La obediencia a las constituciones y estatutos era el primer principio que debían aprender los colegiales. El respeto a los colegiales más antiguos y el orden y la jerarquía interna eran considerados como imprescindibles para el buen gobierno de la casa. Los colegiales más antiguos pronto se encargaron de hacer prevalecer su veterana estancia frente a la inexperiencia de los colegiales noveles. Se reprendía cualquier falta que pudiera perjudicar al resto de colegiales y al honor de la casa. (Martín Sánchez, 2009, p. 379)

En efecto, para nuestro caso, nos permitimos recordar que la Orden de Predicadores tenía tres géneros de casas de estudio (studia): Conventuales, Solemnes (o provinciales) y Generales (universitarios) (Barrado Barquila, 1990). El Estudio General de la provincia dominica nació en su Convento de Santafé, fundado en 1550 y nombrado como Convento de Nuestra Señora del Rosario, estaba ubicado muy cerca de la plaza Mayor. Para el caso de los colegios que se elevaban a universidad debían, en el caso de los dominicos, ser reconocidos por los Capítulos Generales, que aprobaban la erección de un estudio solemne en un convento, después solicitaban del papa una bula para conferir grados, quedando convertidos en Estudio General. En ellos ya no existe una corporación de escolares o graduados como en los primeros tiempos, o al menos 
es solo simbólica (Peset \& García Trobat, 2013). De esta manera, se podían abrir universidades sin contar con la inversión del tesoro real, lo cual, en ocasiones, facilitaba la obtención de la Cédula Real aprobatoria de la nueva universidad. Es de anotar que, para el caso de la Nueva Granada, ni indígena, ni mulato, ni mestizo, ni recién convertido, ni descendiente de judío o árabe o con una vida laboral y jurídica poco destacable eran admitidos a la universidad. Finalmente, se aseguraba que el aspirante no tuviera — ni él o en su familia - enfermedad habitual ni de mal contagioso (Castro-Gómez, 2005, p. 120), lo cual implicaba necesariamente el ser rechazado con el respectivo desprecio social.

Sin embargo, como informa Plata Quezada, los frailes predicadores aceptaron algunos indígenas donados que se ocuparon como legos, aparte de la excepcional circunstancia de fray Martín de Porres. Se conoce el caso de fray Sebastián del Rosario, indígena de Tocancipá, que, a pesar de ser analfabeta, logró tener licencia para predicar a su propia comunidad indígena; la autorización fue otorgada directamente por fray Cristóbal de Torres. Fray Pablo de Meneses, indígena muisca, quien interpretaba el órgano, recibió el hábito de religioso lego, estudió Gramática Latina, oyó Artes y Teología, pero no consiguió licencia para ser sacerdote. Fray Juan de Santo Domingo indio de nación (muisca) tampoco hizo profesión religiosa, ni fray Pedro de Cucunubá, religioso donado quien tuvo una vida ejemplar (Plata Quezada, 2012, p. 95). Como podemos ver, el debate era muy fuerte entre los que estaban a favor o en contra de que se admitieran manchados, como nos ilustra la siguiente cita

Las corrientes en contra del clero indígena arreciaron de nuevo organismo máximo de la Orden de Predicadores cediera. En 1647, el Capítulo General de los dominicos, celebrado en Valencia, España, ordenó, no sólo que no se debía recibir al hábito a los indígenas, sino además a los mestizos y a los mulatos, grupos sociales cada vez más numerosos en América. La 
exclusión debía observarse hasta la cuarta generación inclusive. (Plata Quezada, 2012, p. 96)

Finalmente, para el caso de la Nueva Granada en términos educativos, podemos decir, con García Sánchez, que existían escuelas de primeras letras; escuelas pías; escuelas de lengua castellana y de religión para indios (también conocidas como doctrineras); escuelas dominicales, de encomenderos, y, desde el punto de vista de la gratuidad, gratuitas o pensionarias (García Sánchez, 2005):

También vimos y pulzamos las aulas todas, y (h)alle mucho esmero, y cuidado; haviendo poco el gozo, que he tenido al ver las aulas de Gramática tan poblada de estudiantes, y el esmero con que R. (Reverendo) P. (Padre) Presbítero los enseña, a este le doy las devidas gracias, $\mathrm{Y}$ le encargo prosiga con el mismo cuidado, enseñándolos, no solo en la latinidad, sino también en temor a Dios, que es lo principal, el modo de tratarse por políticamente que oygan misas, que ayuden, como haigan (hayan) de traher el vestido, como hayan de traer el cuerpo, y demás acciones que aunque parezcan materialidades, vienen hacer el molde en donde se forman estos niños ya para servir, y ministrar los altares de Dios; y ya para seguir, governar una República. Para cuyo cumplimiento imploro la vigilancia del R. (Reverendo) P. (Padre) Regente y Vicario Provincial. (AHCSD 1771-1790, f. 213) (Alzate Montes, 2012, p. 61)

Como podemos apreciar, al terminar las primeras letras se pasaba a la cátedra de Gramática, lo cual vendría a ser como el bachillerato hoy en día; este estudio se basaba en el trivium (gramática, retórica y dialéctica); el segundo ciclo, el quadrivium, lo conformaban aritmética, música, geometría y astronomía, todo bajo la Ratio Studiorum, que procedía de la Universidad de París y era adaptado por la orden dominicana (USTA, 1980). 
También existían, para la educación más avanzada, los colegios mayores y los seminarios, que tenían anexas escuelas de diferente naturaleza. Los Colegios Mayores más destacados estaban ubicados en Santafé, Tunja, Popayán, Pamplona, Cartagena y Mérida.

\section{La Tomística}

El primero que pensó fundar un colegio en la Nueva Granada fue el obispo de Cartagena, don fray Jerónimo de Loaiza, pero fue nombrado arzobispo de Lima en 1542, dejando el proyecto inconcluso. Propio de su carisma, los dominicos en su convento impartían clases en su estudio para la formación de sus frailes; es así como la primera cátedra de gramática en Santafé empezó a dictarse en el año de 1563. En 1573 se dieron las primeras clases de filosofía y teología. El primer regente de estudios del Estudio de Santafé fue el padre Alberto Pedrero.

En 1571, el Capítulo General de Avignon había decretado que se erigiera en la provincia de San Antonino un Estudio General con el fin de aprovechar la experiencia de los dominicos en México y Lima; siguiendo esta solicitud, elevan ante su real majestad, el rey don Felipe II, la solicitud para fundar una Universidad en el Convento de Santo Domingo. El encargado de presentar la solicitud fue el procurador de la provincia, padre Juan Méndez; sin embargo, la respuesta del rey, el 10 de noviembre de 1573, fue una serie de preguntas a la Real Audiencia sobre la conveniencia para crearse una universidad en el convento del Rosario en Santafé (USTA, 1980), la cual fue respondida favorablemente por fray Pedro Bedón el 6 de abril de 1595. Ahora bien, de acuerdo con José Abel Salazar (USTA, 1980), los dominicos tomaron por Real Cédula la comunicación del rey, lo cual fue motivo de protesta por parte de los jesuitas. Entre tanto, fray Francisco de Carvajal, en el Capítulo General de Roma en 1580, logra el apoyo del maestro 
general, fray Paulo Constable para que en el convento haya un Estudio General como Universidad ${ }^{4}$ y, a su vez, que haga la solicitud al papa para la creación de un Estudio General, obteniendo como respuesta la bula Romanus Pontifex del 13 de junio del mismo año, con especiales gracias donadas por el sumo pontífice:

- Que el convento del Rosario de Santa Fe se elija como Universidad de Estudios Generales.

- Con rector, lectores y con las facultades y concesiones acostumbradas en la Orden de Predicadores.

- Que puedan los alumnos ganar sus cursos, ser graduados de bachiller, licenciados, maestros y doctores por los rectores de dicha universidad, o por los maestros deputados para ello

-Y los graduados disfruten de todas las gracias, privilegios, favores, y prerrogativas de que gozan los graduados de cualquier universidad en España (USTA, 1980).

Así las cosas, en 1608 se consideraba cabalmente Universidad a aquella institución que contaba con todos los documentos pontificios y reales (de ahí el nombre Real y Pontificia); pero este no era el caso de la Tomística, ya que el permiso pontificio en algún momento se perdió y se puso en duda su veracidad y, de otra parte, tenía un documento controvertible que le acreditaba como universidad regia, es decir, que los dos documentos podrían ser impugnables. A esto hay que sumarle que, a causa del incidente con la bula Sublimis Deus - que le costó la cárcel a fray Bernardino de

4 "In Provincia Sancti Antonini de Novo Regno Indiarum maris Oceani acrptamos et reigimus in Universitatem Conventaum Sanctae Mariae del Rosario in Civitate de Santa Fé cum iiusdem gratiis et privilegiis consuetis". 
Minaya-, el rey Carlos V ordenó que, en adelante, "todas las Bulas para Indias se impetraran a través suyo, so pena de nulidad" (González González, 2015, p. 29) y, en septiembre de 1538, expidió una célula que invalidaba todas las bulas que circulaban sin su aval expreso: el llamado para ser real, ahora bien, se deduce por lo anteriormente dicho, que la bula de Gregorio XIII no contaba con el aval del Patronato Regio (González González, 2015 , p. 30).

Es así como la universidad tuvo que esperar a que se fundara el colegio Santo Tomás, gracias a la donación de Gaspar Núñez, la cual contaba con una dotación cercana a los 30000 pesos: "ante el escribano Alonso de Torralva los prendados de la provincia dominicana aceptaron las dotación y fundación del colegio titulado Santo Tomás del Rosario, y el 11 de mayo del mismo año 1608, tomaron posesión de él. Y al mes de julio siguiente la provincia de San Antonino se dio los privilegios universitarios del convento santafereño al nuevo colegio" (USTA, 1980, p. 141). La autorización papal para que los derechos universitarios pasaran del convento a la universidad se lograron a través de la gestión de Antonio Biedma y Melchor Núñez en la bula Cathedram Militantes Ecclesiae de 1612, pero solo hasta 1619, con el breve Charissimi in Christo, el papa autoriza a la Tomística otorgar títulos. Es a partir de este breve que el rey otorga la Cédula el 6 de septiembre de 1624, pero solo hasta el 26 de abril de 1626 el presidente de la Real Audiencia de Santafé le dio cumplimiento. Desde ese año, la Tomística fue reconocida como Colegio-Universidad (menor); sus títulos universitarios solo eran válidos en las Indias y su sostenimiento era responsabilidad total de la Orden de los Frailes Predicadores (Restrepo Zea, 1991).

En medio de todas estas circunstancias que impidieron su desarrollo normalmente, además de los pleitos con los jesuitas, el trámite para pasar los derechos de universidad del convento al Colegio y las disputas intestinas en torno al tema de la admisión de indios, mestizos y mulatos, llegó la nueva institución a entrar en funcionamiento, no como Convento-Universidad, sino como 
Colegio-Universidad, inaugurándose el día 8 de julio de 1639 a manos de don Alfonso de la Cadena y Sandoval, don Martín de Saavedra y Guzmán, presidente del Nuevo Reino y otras personalidades; acto seguido, fray Francisco Farfán disertó sobre la cuestión Utrum beatitudo consistat in potestae. Después de la procesión, se dio por terminado el acto y se reconoció públicamente la validez de la universidad. Este acto permitía que el canciller - un dominico - otorgara los grados y ya no el arzobispo, tal como el modelo salamantino exigía.

Es de singular atención que el día 4 de agosto de 1639 empezó la Tomística sus actividades con los siguientes maestros: rector vitalicio, Bartolomé Núñez; regente de estudios, Francisco de San Pedro; catedrático de prima teología, Francisco Farfán; catedrático de vísperas, fray Tomás Navarro; maestro de estudiantes, Francisco Suárez; catedrático de artes, Marcos de Rojas; Tomás Noguera, catedrático de gramática; el padre Alonso Ronquillo de lengua indígena ${ }^{5}$. Para el año de 1644, el Capítulo General de Roma corroboró las fundaciones y ratificó de rector a Bartolomé Núñez.

En cuanto a los estatutos y ordenaciones, la documentación puede resultar confusa porque en ella se reúnen, en diferentes momentos, los estatutos del convento, del colegio y de la universidad, pero a grandes trazas podemos señalar que en los primeros estatutos estuvo involucrado el maestro Agustín Galamini, entonces maestro general de la Orden (aprobados en París en el año 1611). Los primeros reglamentos estuvieron a cargo de los padres Francisco de la Cruz y Francisco Suárez. En cuanto a los estatutos de

5 Es de anotar que Ronquillo, junto a fray Diego de Valverde y fray Juan Martínez, fue examinador de la Gramática de Fray Bernardo de Lugo; pues desde el III Concilio de Lima (1583) se acordó que la evangelización se hiciera en lengua indígena. Pero en 1770, Carlos III impuso la enseñanza de la doctrina cristiana en castellano, terminando con el proyecto de evangelización en la lengua indígena. Fuente: Boletín del Instituto Caro y Cuervo, Tomo xxxiI septiembre-diciembre de 1977, Número 3. 
la universidad, fueron ordenados por el arzobispo salamantino Fernando Arias de Ugartey y aprobados por el presidente de la Real Audiencia, Juan de Borja, el 1 de abril de 1626, los cuales estuvieron vigentes hasta 1639, pero debido al cambio de Cancelario a manos de la Orden, los nuevos estatutos fueron redactados por Francisco de la Cruz y entraron en vigor con la inauguración de la universidad, manteniéndose hasta 1658 (USTA, 1980, p. 167). Años después, debido a los pleitos jurídicos, las autoridades solicitan los estatutos vigentes, pero, debido al incendio de 1661, los originales se perdieron para siempre; ante esta situación, los padres dominicos acuden a los estatutos de la universidad de Ávila como propios. Esta situación resultó inaceptable, por lo cual la Real Audiencia, en 1770, les ordena seguir los estatutos salamantinos de la universidad de Lima. Aquí es donde la Universidad de Estudio General dominicana se torna mixta, pues, aunque su estructura pertenece al modelo de formación conventual de Alcalá, a partir de 1770, como colegiada, asume el espíritu académico de la universidad contrarreformista de Salamanca.

A pesar de todos estos cambios, la Universidad se mantuvo fiel al escolasticismo peripatético, se opuso a Copérnico (célebre es la disputa con Mutis) y mantuvo la Ratio Studiorum como horizonte formativo:

Para los profesores el currículo completo de sus estudios comprende ocho años de los cuales los dos primeros se asignan a las disciplinas filosóficas y a los elementos de las ciencias naturales y los otros seis a la Teología. Los estudiantes de Filosofía asistirán a dos clases de hora y media todos los días no feriados, en la clase de la mañana se les darán lecciones de lógica, Metafísica y Ética, con algún apéndice de Historia de la Filosofía en cuanto lo permita el tiempo; en la clase de la tarde elementos de Matemáticas o sea Aritmética, Geometría, Álgebra y de Física general y experimental. 
Los demás que se hayan mostrado suficiente mente dotados de talento y de amor al estudio, y también de ardiente celo de la vida religiosa y hayan dado pruebas de actitudes suficientes para el cargo de Lector, en lo sucesivo, emprenderán el estudio de la Suma del Divino Tomás nuestro Preceptor, de la cual tendrán dos clases diarias durante cuatro años juntamente con una de Sagrada Escritura, tantas veces recomendada en los Capítulos Generales.

Cumplidos los años en el estudio del Divino Tomás solamente quienes hayan sido aprobados después de un examen anual, comenzarán a gozar durante los dos últimos años del título y los privilegios de los colegiales ${ }^{6}$.

De igual modo, se mantuvo el modelo medieval de la cátedra de gramática y latinidad; se siguió religiosamente la doctrina de Santo Tomás, lo cual fue una de las quejas de Moreno y Escandón, quien expresaba que "basta decir que los jóvenes de mejor esperanza, no teniendo en que ejercitar su talento, aspiran como precisados a obtener un curato, en que apartados del trato y comercio civil, abandonando el estudio viven como idiotas, por el contrario sería digno objeto de la admiración de los literatos" (USTA, 1980, p. 182). En la Tomística se defendían con fiereza los dogmas de la iglesia, como, por ejemplo, el dogma de la inmaculada concepción. En síntesis, la universidad se había especializado en los saberes universales de la filosofía y la teología y se había dejado para un segundo plano los conocimientos particulares (Barrado Barquilla, 1995, p. 258); los cursos se extendían desde el 18 de octubre hasta el 24 de junio, como ocurría en España:

6 De las Constituciones, Declaraciones y Ordenanzas Capítulo General dadas desde el año 1220 hasta el año 1659 publicadas y organizadas por el reverendísimo padre Fr. Vicente Fontana y divulgadas en 1655, continuadas hasta 1862. [Traducción Dayan Herrera Bernal]. 
11. Que de ninguna manera se expongan en las Tesis una propuesta que pueda ser motivo de ofensa para el poder eclesiástico o laico. Roma 1777 acerca de los estudios. Ordenación 9. En las Tesis que suelen proponerse, no solamente en público sino también en privado, para ejercitar el ingenio, de ningún modo se exponga una propuesta que pueda ser ofensiva para el poder eclesiástico o laico; y que no se aceptan controversias de las que puedan surgir mal entendidos para la Orden (...)

15 Y que nadie en adelante pueda recibir el título de Predicador si no haya completado el curso de Artes y, no haya asistido por un trienio al curso de teología?

Es de anotar que hubo Estudios dominicanos que fueron elevados a Estudio General afiliados a la Tomística, como fue el caso de Tunja (1608), que se organizó por lo menos diez años antes que el de Santafé; además, están el de Cartagena (1644), Santa Marta (1673), el Colegio de Mérida de Maracaibo (1803) y el Seminario de Caracas (1806). Los Colegios de Popayán, San Bartolomé y el Rosario de Santafé también estuvieron vinculados en algún momento.

El cierre de la universidad se dio el 5 de noviembre de 1861, por orden de Tomás Cipriano de Mosquera. La Universidad Santo Tomás permaneció cerrada cerca de cien años, por lo que los frailes dominicos volvieron a organizarse en el país hasta el siglo xx y, como ha sido su historia, en medio de turbulencias, logró restaurarse. Su puesta en marcha como Universidad se logró finalmente el 7 de marzo de 1965, con 12 directivos, 33 profesores y 273 alumnos, en cuatro facultades: Ingeniería Civil, Economía y Administración de Empresas; Filosofía y Ciencias Jurídicas (Derecho); y Filosofía y Ciencias Sociales (Sociología) (Gómez Bello, 2015).

7 Traducción de Dayan Herrera Bernal. 


\section{Conclusiones}

Hemos podido constatar a lo largo de cuatro siglos cómo la universidad se ha adaptado con fidelidad creativa a su impronta dominicana y humanista. De esta adaptación ha surgido una universidad que se caracteriza por su capacidad de transformación a las nuevas vicisitudes que los tiempos le han impuesto. También hemos podido apreciar que, a causa de los trámites legales de la época, la Universidad Santo Tomás se ha convertido en un modelo excepcional de universidad que recoge lo mejor de las dos tradiciones que la nutrieron y que en razón a ello ha modificado su fisonomía hasta convertirse en una universidad madura, que con su singular personalidad se distingue de otros modelos universitarios.

\section{Referencias}

Aquino, T. (1994). Suma teológica. Madrid: Biblioteca de autores Cristianos.

Alzate Montes, C. M. (2012). Diario de un convento. Santo domingo de Tunja durante la Independencia. Bogotá: Ediciones UsTA.

Arredondo Vega, D. M. (2011). Los modelos clásicos de universidad pública el 4 de noviembre de 2016, de Odiseo, Revista electrónica de Pedagogía: http://www.odiseo.com.mx/articulos/ modelos-clasicos-universidad-publica

Barcala Muñoz, A. (1985). Las universidades españolas durante la Edad Media. Anuario de estudios medievales, 15, 83-126.

Barrado Barquila, J. (1990). Actas del II Congreso Internacional sobre los Dominicosy el Nuevo Mundo, Salamanca, 28 de marzo-1 de abril de 1989 (Vol. 2). J. Barrado Barquila, Ed.) Madrid: San Esteban.

Barrado Barquilla, J. (1995). Los Dominicos y el Nuevo Mundo, siglos XVIII-XIX: actas del IV Congreso Internacional, Santafé de Bogotá, 6-10 septiembre 1993. Madrid: Editorial San Esteban. 
Beuchot, M. (1991). El realismo cognoscitivo en Santo Tomás de Aquino. Sus condiciones metafisicas. Recuperado el 16 de enero de 2016, de Diánoia volumen XXXVII(37). Obtenido de http://www.dominicos.org/espiritualidad/ dominicana/textos/galeria-de-personajes/vicente-de-couesnongle(14 de 08 de 2016)

Castro-Gómez, S. (2005). La hybris del punto cero: ciencia, raza e ilustración en la Nueva Granada (1750-1816). Bogotá: Editorial Pontificia Universidad Javeriana.

Espeja Pardo, J. O. P. (2016). Misión, individuo y comunidad el dinamismo de la espiritualidad dominicana. Recuperado el 7 de enero de 2016, de dominicos.org: Http://www.dominicos.org/kit_upload/file/Espiritualidad/ Mision-Espiritualidad-del-fraile-dominico-Jesus-Espeja.pdf

García Sánchez, B. Y. (2005). La educación colonial en la Nueva Granada: entre lo doméstico y lo público. Historia de la educación latinoamericana. (Rhela), 7, 217-238.

Gómez Bello, E. (2015). Desde la restauración hasta la Usta Colombia. En usta, De la tomística de Santafé a la Universidad Santo Tomás de Colombia en los últimos 50 años (p. 305). Bogotá: Ediciones UsTA.

Gómez, E. \& Moreno, M. (2015). Filosofia y cultura Institucional. Bogotá: Ediciones USTA.

González González, E. (2015 ). En busca de universidad. Santo Domingo, México y Lima en el siglo XVI. Universidades (LXVI), 23-40.

Hernández González, M. V. y Casado Arboniés, M. (2004). Estudiantes Canariots en las universidades de Alcalá y Sigüenza durante el Antiguo régimen y su proyección en América. Revista de Historia Canaria, 186, 119-153.

Historia de la Facultad de Teología (28 de 11 de 2016). Recuperado el 28 de 12 de 2016, de Facultad de Teología San Esteban: http://www.fatse.org/ facultad/historia

Jiménez Moreno, L. (1996). La Universidad Complutense Cisneriana: impulse filosófico, científico y literario siglos XVI y XVII. Madrid: Editorial Complutense.

Magallón Ibarra, J. M. (2002). El renacimiento medieval de la jurisprudencia romana. México: Universidad Nacional Autóna de México. 
Marquínez Argote, G. (2001). La escolástica colonial de los siglos XVI-XVII. En: G. Marquínez Argote, L. J., González Álvarez, J., Zabalza Iriarte, J. A., Suárez Alarcón \& G. M. Argote (Ed.), La filosofia en América Latina: historia de las ideas. (p. 81). Bogotá: El Búho.

Martín Hernández, F. (1992). La influencia de los Colegios Mayores españoles en la fundación y primer desarrollo de los americanos. (n. p., Trad.) Estudios de historia social y económica de América(9), 9-22.

Martín Sánchez, M. A. (2009). La formación clerical en los colegios menores de la Universidad de Salamanca. (4), 367-389.

Migueláñez, G. P. (2011). Cultura y humanismo en la América colonial española (Vol. 1). Libros en Red.

Peset, M. \& García Trobat, P. (2013). El nacimiento de la primera Universidad de la Compañía de Jesús. Revista Borja(34), 107-129.

Peset Reig, M. (2015). Modelos historiográficos de las primeras universidades. Universidades, LXVI(65).

Plata Quezada, W. E. (2012). Vida y muerte de un convento. Domincios y sociedad en Santafé de Bogotá (Colombia) siglos XVI-XIX. (p. 96, Trad.) Madrid: Editorial San Esteban.

Restrepo Zea, S. (1991). La educación de el Nuevo Reino de Granada durante el siglo XVII. Revista Educación y Pedagogía (6).

Rodríguez Cruz, Á. (1998). Análisis comparativo de las constituciones universitarias de Salamanca con las hispoanoamericanas. Estudios de historia social y económica de América (16-17), 583-591.

Rodríguez Cruz, Á. (2008). Protagonismo de la Universidad de Salamanca en los pensadores y forjadores de las universidades hispanoamericanas. En Unesco, Pensadores y forjadores de la universidad latinoamericana (pp. 57 102). Caracas: Unesco / Iesalc - Cendes, Bid\&Co.

Santiago-Otero, H. (1996). Los "Estudios Generales" en Hispania y la fundación del "Estudio" de Alcalá de Henares. En: L. J. Moreno, La Universidad Complutense Cisneriana: impulse filosófico, científico y literario siglos XVI y XVII (pp. 45-64). Madrid: Editorial Complutense.

Sedano González, J. d. (2002). Pedagogía de la respuesta: horizontes para la formación humana. Bucaramanga: Convento de Cristo Rey. 
Tünnermann, B. C. (1996). Historia de la universiad en América Latina. De la época colonial a la Reforma de Córdoba. Caracas: CREsAla.

Tünnermann Bernheim, C. (1996). La educación superior en el umbral del siglo XXI. Caracas: cresalc.

usta (2010). Dimensión de la política docente. Bogotá: Ediciones usta.

USTA (2015). Documento marco gestión del talento humano. Bogotá: Ediciones USTA.

usta (2004). Estatuto Ordenado. Bogotá: Ediciones usta.

usta (2010). Informe Ejecutivo de Acreditación. Bogotá: Ediciones usta.

usta (2004). PEI. Bogotá: Ediciones USTA.

usta (2009). Política de investigación. Bogotá: Ediciones Usta.

usta (1980). Universidad Santo Tomás, 400 años. Bogotá: Centro de enseñanza desescolarizada.

Woods, J. T. (31 de diciembre de 2005). La Iglesia católica y la creación de la Universidad. Obtenido de Centro de Recursos para la Universidad Católica: http://www.catholiceducation.org/es/educacion/aportes-del-catolicismo/la-iglesia-catolica-y-la-creacion-de-la-universidad.html 
